\title{
On Spinor Varieties and Their Secants ${ }^{\star}$
}

\author{
Laurent MANIVEL
}

Institut Fourier, Université de Grenoble I et CNRS, BP 74, 38402 Saint-Martin d'Hères, France

E-mail: Laurent.Manivel@ujf-grenoble.fr

URL: http://www-fourier.ujf-grenoble.fr/ manivel/

Received April 03, 2009, in final form July 21, 2009; Published online July 24, 2009

doi:10.3842/SIGMA.2009.078

\begin{abstract}
We study the secant variety of the spinor variety, focusing on its equations of degree three and four. We show that in type $D_{n}$, cubic equations exist if and only if $n \geq 9$. In general the ideal has generators in degrees at least three and four. Finally we observe that the other Freudenthal varieties exhibit strikingly similar behaviors.
\end{abstract}

Key words: spinor variety; spin representation; secant variety; Freudenthal variety

2000 Mathematics Subject Classification: 14M17; 15A66; 14L35; $14 \mathrm{~N} 15$

\section{Introduction}

This paper grew out from a striking observation in a preprint version of [13], according to which the spinor variety of type $D_{7}$ was the unique compact Hermitian symmetric space whose secant variety (with respect to its minimal equivariant embedding) had no cubic equation.

Among the most familiar Hermitian symmetric spaces, the Scorza varieties, which parametrize matrices (possibly symmetric or skew-symmetric) of rank one (up to scalar), have extremely wellbehaved secant varieties: the $k$-th secant $\sigma_{k}(X)$ of a Scorza variety $X$ parametrizes matrices of rank at most $k+1$, and its ideal is generated by explicit equations of degree $k+2$, given by minors and their generalizations (a standard reference for secant varieties, including Scorza varieties, is [15]). In particular the first secant variety $\sigma(X)$ is cut-out by cubics, and it was tempting to think that $\sigma(X)$, for many equivariantly embedded rational homogeneous spaces, or at least for the simplest ones, should have many cubic equations. Moreover, writing down such equations explicitly would be a step towards a generalized theory of minors.

The class of Hermitian symmetric spaces (possibly enlarged to the so-called cominuscule varieties) being particularly well-behaved, it was tempting to try to understand better the observation of [13] about the spinor variety of type $D_{7}$. Our first aim was to understand, for a spinor variety $S$ of type $D_{n}$, the cubic equations of $\sigma(S)$. The answer to this problem is essentially given by Theorem 2, where we provide the decomposition into irreducibles of the symmetric cube of a half-spin representation, combined with Theorem 3. Our conclusion is that there are no cubic equations for the secant variety exactly in types $D_{7}$ and $D_{8}$. In type $D_{9}$ there exists a family a cubic equations parametrized by the other half-spin representation.

On the other hand quartic equations exist already in type $D_{7}$ (in type $D_{6}$ and lower, the secant variety is the whole ambient space). Theorem 4 allows a partial understanding of quartic equations of $\sigma(S)$. In particular, in any type $D_{n}$, with $n \geq 7$, there exist quartic equations which cannot be derived from cubic ones.

Finally, in the last sections we put our results in a broader perspective. The point is that spinor varieties are only one class among the so-called Freudenthal varieties, which also include Lagrangian Grassmannians, and Grassmannians of middle-dimensional subspaces of an even

\footnotetext{
*This paper is a contribution to the Special Issue "Élie Cartan and Differential Geometry". The full collection is available at http://www.emis.de/journals/SIGMA/Cartan.html
} 
dimensional vector space. It turns out that at least with respect to their cubic and quartic equations, Freudenthal varieties exhibit a strikingly uniform behavior. It would be interesting to understand better these similarities and their limits.

\section{Spinor varieties and Pfaffians}

It was already observed by Chevalley that an open subset of the spinor variety can be parametrized by Pfaffians; more precisely, by the complete set of sub-Pfaffians of a generic skew-symmetric matrix. An interesting consequence is that the equations of the spinor variety provide polynomial relations between these sub-Pfaffians. Such relations have been known from the very beginning of Pfaffian theory, the most famous one being the rule analogous to the line or column expansion of determinants. Many other such relations have been found, including Tanner's relations, and its relatively recent generalization by Wenzel ([4], see also [9] for a short proof).

The link with spinor varieties has the following immediate consequences. First, since the ideal of the spinor variety is generated in degree two by Kostant's theorem, it follows that all the algebraic relations between sub-Pfaffians are generated by quadratic relations. Second, these quadratic relations can be completely explicited, using the full set of quadratic equations for the spinor variety obtained by Chevalley. Since these equations are more convenient to express in terms of Clifford algebras, we will begin with a brief review of the tools involved. Our basic reference is [3].

\subsection{Clifford algebras and spin representations}

Let $V$ be a complex vector space of dimension $2 n$, endowed with a non degenerate quadratic form $q$. Suppose that $V=E \oplus F$ splits as the direct sum of two maximal isotropic vector spaces, of dimension $n$. For any $v \in V$, we can define two natural operators on the exterior algebra $\wedge V$. One is the exterior product $o(v)$ by $v$; the other one is the inner product $i(v)$ defined by the contraction with $v$ through the quadratic form; both are graded operators, of respective degree +1 and -1 .

Let $\psi(v)=o(v)+i(v)$. An easy computation shows that the map $\psi: V \rightarrow \operatorname{End}(\wedge V)$ extends to the Clifford algebra $C l(V)$, the quotient of the tensor algebra of $V$ by the relations

$$
v \otimes w+w \otimes v=2 q(v, w) 1
$$

Applying to $1 \in \wedge^{0} V$, one obtains a vector space isomorphism

$$
\theta: C l(V) \simeq \wedge V
$$

which is compatible with the action of $V$ on both sides. In particular the inverse isomorphism sends $v \wedge w \in \wedge^{2} V$ to $v w-q(v, w) 1 \in C l(V)$. Note that $\wedge^{2} V$ is naturally isomorphic to $\mathfrak{s o}(V)$ and that the resulting map $\mathfrak{s o}(V) \rightarrow C l(V)$ is a morphism of Lie algebras, once the associative algebra $C l(V)$ has been endowed with its natural Lie algebra structure.

Let $f \in C l(V)$ be the product of the vectors in some basis of $F$ ( $f$ is well defined up to a non zero scalar). Then $C l(V) f$ is a left ideal of $C l(V)$ isomorphic, as a vector space, to $\wedge E$ (this is because $C l(V) \simeq C l(E) \otimes C l(F)$ and $C l(F) f=\langle f\rangle)$. This makes of $\wedge E$ a $C l(V)$-module, with an action of $v=v^{\prime}+v^{\prime \prime} \in V=E \oplus F$ defined by $o\left(v^{\prime}\right)+2 i\left(v^{\prime \prime}\right)$ (beware to the factor 2 here!).

The spin group $\operatorname{Spin}(V)$ is defined as a subgroup of the group of invertible elements in the Clifford algebra. In particular any $C l(V)$-module is also a $\operatorname{Spin}(V)$-module. As a $\operatorname{Spin}(V)$ module, $\wedge E$ splits as the direct sum of the two half-spin representations $\wedge^{\text {odd }} E$ and $\wedge^{\text {even }} E$, both of dimension $2^{n-1}$, that we will also denote $\Delta_{+}$and $\Delta_{-}$. A priori these two representations 
cannot be distinguished. Our convention will be the following: if $\epsilon(n)$ is the parity of $n$, then we let

$$
\Delta_{+}=\wedge^{\epsilon(n)} E .
$$

This will have the advantage that the pure spinor defined by $E$ will belong to the projectivization of $\Delta_{+}$.

\subsection{Pure spinors}

The variety of maximal isotropic subspaces of $V$ has two connected components $S_{+}$and $S_{-}$, which are (non canonically) isomorphic. Their linear spans in the Plücker embedding are in direct sum: there is a splitting

$$
\wedge^{n} V=\wedge^{n} V_{+} \oplus \wedge^{n} V_{-}
$$

into spaces of the same dimension, and $S_{ \pm}=G(n, 2 n) \cap \mathbb{P}\left(\wedge^{n} V_{ \pm}\right)$are linear sections of the usual Grassmannian. But the minimal embeddings of $S_{+}$and $S_{-}$are embeddings in the projectivized half-spin representations: $S_{+}$is the $\operatorname{Spin}(V)$-orbit, in the projectivization of $\Delta_{+}$, of the line $\wedge^{\text {top }} E=\left\langle e_{1} \wedge \cdots \wedge e_{n}\right\rangle$, where $e_{1}, \ldots, e_{n}$ is a basis of $E$. The embedded varieties

$$
S_{+} \subset \mathbb{P} \Delta_{+} \quad \text { and } \quad S_{-} \subset \mathbb{P} \Delta_{-}
$$

are called the varieties of even and odd pure spinors, respectively. The more familiar embeddings into $\mathbb{P}\left(\wedge^{n} V_{ \pm}\right)$can be recovered from these minimal embeddings through a quadratic Veronese map.

Two maximal isotropic subspaces of $V$ are in the same spinor variety if and only if the dimension of their intersection has the same parity as $n$. In particular $E$ and $F$ belong to the same family if and only if $n$ is even.

If $H \subset V$ is any maximal isotropic subspace, its representative in $S_{+} \cup S_{-}$can be obtained as follows. Let $h \in C l(V)$ be the product of the vectors of a basis of $H$. Then the left ideal $C l(V) f$ intersects the right ideal $h C l(V)$ along a line, which can be written as $u_{H} f$ for a unique line $u_{H} \in \mathbb{P}(\wedge E)$. According to the parity of the dimension of $H \cap E$ one gets in fact a line $u_{H} \in \mathbb{P}\left(\wedge^{ \pm} E\right)=\mathbb{P} \Delta_{ \pm}$, representing the point of the spinor variety defined by $H$.

Consider for example, for any subset $I$ of $\{1, \ldots, n\}$, the maximal isotropic space $H_{I}$ generated by the vectors $e_{i}$ for $i \in I$, and $f_{j}$ for $j \notin I$, where $f_{1}, \ldots, f_{n}$ is the basis of $F$ such that $q\left(e_{i}, f_{j}\right)=\delta_{i j}$. Then $u_{H_{I}}$ is the line generated by $e_{I}$, the wedge product of the $e_{i}$ 's, $i \in I$.

Proposition 1. The action of $\operatorname{Spin}(V)$ on $S_{+}$is generically 3-transitive (but not 4-transitive), in the sense that $\operatorname{Spin}(V)$ has an open orbit in $S_{+} \times S_{+} \times S_{+}$.

Proof. We treat the case where $n=2 m$ is even. Then $E$ and $F$ both belong to $S_{+}$, as well as the space $G=\left\langle e_{2 i}+f_{2 i-1}, e_{2 i-1}-f_{2 i}, 1 \leq i \leq m\right\rangle$. The stabilizer of $(E, F)$ in $S_{+} \times S_{+}$is easily seen to be equal to $G L_{n}$, embedded almost diagonally in $S O_{2 n}$ by the morphism $A \mapsto$ $\left(A,{ }^{t} A^{-1}\right) \in G L(E) \times G L(F) \subset G L(E \oplus F)$. The additional condition that $G$ be preserved is then equivalent to the condition that $A$ preserved the symplectic form $\omega$ on $E$ defined by $\omega\left(e_{2 i}, e_{2 i-1}\right)=1$ and $\omega\left(e_{j}, e_{k}\right)=0$ if $j, k$ is not of the form $2 i, 2 i-1$. So the stabilizer of the triple $(E, F, G) \in S_{+} \times S_{+} \times S_{+}$is a copy of the symplectic group $S p_{n}$ inside $S O_{2 n}$. In particular the orbit of $(E, F, G)$ inside $S_{+} \times S_{+} \times S_{+}$has dimension $\operatorname{dim} S O_{2 n}-\operatorname{dim} S p_{n}=3 \operatorname{dim} S_{+}$, so it must be dense.

The smooth varieties $S_{+}, S_{+} \times S_{+}, S_{+} \times S_{+} \times S_{+}$are thus smooth compactifications of the homogeneous spaces $S O_{n}, S O_{2 n} / G L_{n}$ and $S O_{2 n} / S p_{n}$, respectively. 
We thus recover the fact (which holds for any rational homogeneous variety, equivariantly embedded) that the secant variety $\sigma\left(S_{+}\right)$to the spinor variety is quasi-homogeneous. However the second secant variety $\sigma_{2}\left(S_{+}\right)$is not, because the stabilizer $S p_{n}$ of a general triple in $S_{+}$acts trivially on the corresponding lines of $\Delta_{+}$. So a general point of $\sigma_{2}\left(S_{+}\right)$can be put, up to the group action, in the form $t_{E} u_{E}+t_{F} u_{F}+t_{G} u_{G}$, but the triple $\left(t_{E}, t_{F}, t_{G}\right) \in \mathbb{P}^{2}$ cannot be reduced to $(1,1,1)$.

For future use we choose the following general points of $S_{+} \times S_{+}$and $S_{+} \times S_{+} \times S_{+}$:

If $n=2 m$ is even, $E$ and $F$ belong to $S_{+}$, and $G$ defined above as well. Their representatives in $\mathbb{P} \Delta_{+}$are

$$
u_{E}=e_{1} \wedge \cdots \wedge e_{n}, \quad u_{F}=1, \quad u_{G}=\wedge_{i=1}^{m}\left(1+e_{2 i-1} \wedge e_{2 i}\right) .
$$

If $n=2 m+1$ is odd, $E$ belongs to $S_{+}$but $F$ belongs to $S_{-}$, so we denote by $F^{\prime}$ the maximal isotropic subspace generated by $f_{1}, \ldots, f_{n-1}, e_{n}$, which belongs to $S_{+}$. Then

$$
u_{E}=e_{1} \wedge \cdots \wedge e_{n}, \quad u_{F^{\prime}}=e_{n} .
$$

The sum $u_{E}+u_{F}$ (respectively $u_{E}+u_{F^{\prime}}$ ) defines a generic point of the secant variety $\sigma\left(S_{+}\right)$, more precisely a point in the open orbit.

\subsection{Pfaffians}

Pfaffians appear when one tries to parametrize the spinor variety $S_{+}$, at least in a neighborhood of the point defined by $E$. This is exactly similar to the fact that minors of a generic matrix are Plücker coordinates of a generic point of the Grassmannian.

For this, we use our preferred basis $e_{1}, \ldots, e_{n}$ of $E$. Let $u=\left(u_{i j}\right)$ be any skew-symmetric matrix of size $n$. Then the vectors

$$
e_{i}(u)=e_{i}+\sum_{j=1}^{n} u_{i j} f_{j}, \quad 1 \leq i \leq n
$$

generate a maximal isotropic subspace $E(u)$ in the same family as $E$, that is $S_{+}$. A computation yields the following formula for the corresponding pure spinor (this is a slight extension of the way Chevalley defines the Pfaffian in [3, p. 57]):

$$
u_{E(u)}=\sum_{\ell(K) \text { even }} \operatorname{Pf}_{K}(u) e_{K^{c}}
$$

where the sum is over the sequences $K=\left(k_{1} \cdots k_{\ell}\right)$ of integers between 1 and $n$, of even length $\ell=\ell(K), K^{c}$ is the complementary subset of integers, and $\operatorname{Pf}_{K}(u)$ is the Pfaffian of the submatrix of $u$ obtained by taking lines and columns indexed by $K$. Since $E(u)$ is a generic maximal isotropic subspace in the same family as $E$, this formula provides a rational parametrization of a dense open subset of the spinor variety $S_{+}$.

\subsection{Equations}

Which equations characterize pure spinors? Kostant's theorem asserts that, as for any equivariantly embedded rational homogeneous space, the ideal of $S_{+}$is generated in degree two. Moreover, the quadratic equations of $S_{+}$can be written down very explicitly, as follows.

We need to introduce the main anti-automorphism of $C l(V)$, which is characterized by the fact that $\alpha\left(v_{1} \cdots v_{r}\right)=v_{r} \cdots v_{1}$ if the space generated by $v_{1}, \ldots, v_{r}$ is isotropic. Then, for any $u, v \in \Delta_{+} \subset C l(V)$, let

$$
\beta(u, v)=u f \alpha(v) \in C l(V) \simeq \wedge V,
$$


where the isomorphism between $C l(V)$ and $\wedge V$ is defined as above. Denote by $\beta_{k}(u, v)$ the projection of $\beta(u, v)$ to $\wedge^{k} V$. Then

1. $\beta_{k}$ is non zero if and only if $n-k=2 p$ is even;

2. then it is symmetric for $p$ even and skew-symmetric for $p$ odd;

3. $\beta_{k}$ and $\beta_{2 n-k}$ coincide up to sign;

4. $u \in \Delta_{+}$defines a pure spinor if and only if $\beta_{k}(u, u)=0$ for all $k$ smaller than $n$.

Indeed, in case $u$ represents a pure spinor, that is, a maximal isotropic subspace of $V$, one can check that $\beta(u, u)$ is the product of the vectors in a basis of that space. This implies that $\beta_{k}(u, u)=0$ for all $k \neq n$. Conversely, the last assertion is that this property defines $S_{+}$, and more precisely, we get the complete space of quadratic equations of $S_{+}$- whence the whole ideal.

A simple computation yields the following formula, which will be of fundamental importance in the sequel. Recall that we denoted by $F^{\prime}$ the maximal isotropic subspace generated by $f_{1}, \ldots, f_{n-1}, e_{n}$.

Proposition 2. Suppose $n$ is even. Then, up to a non zero constant,

$$
\beta_{2 k}\left(u_{E}, u_{F}\right)=\sum_{\ell(K)=k} e_{K} \wedge f_{K}
$$

Suppose $n$ is odd. Then, up to a non zero constant,

$$
\beta_{2 k+1}\left(u_{E}, u_{F^{\prime}}\right)=\sum_{\ell(K)=k} e_{K} \wedge f_{K} \wedge e_{n}
$$

\subsection{Pfaffian identities}

Applying the previous identities to the generic maximal isotropic subspace $E(u)$ and the corresponding pure spinor, we shall obtain quadratic identities between sub-Pfaffians of a generic skew-symmetric matrix. As explained above, we will obtain all the relations of that kind, and every algebraic relation between these sub-Pfaffians can be deduced from these.

The only computation we need is that of $\beta\left(e_{I}, e_{J}\right)=\psi\left(e_{I} f \alpha\left(e_{J}\right)\right)$.1. Let $f_{1}, \ldots, f_{n}$ be a basis of $F$ such that $q\left(e_{i}, f_{j}\right)=\delta_{i j}$. We can suppose that $f=f_{1} \cdots f_{n}$.

Lemma 1. For any $I, J$, we have

$$
\beta\left(e_{I}, e_{J}\right)=2^{|I \cap J|} \sum_{R \subset I \Delta J} \epsilon(I, J, R) e_{I \cap J} \wedge e_{R} \wedge f_{I^{c} \cap J^{c}} \wedge f_{R}
$$

with $\epsilon(I, J, R)= \pm 1$. (We denoted $I \Delta J=(I \backslash J) \cup(J \backslash I)$ and $I^{c}$ the complement of $\left.I.\right)$

Proof. First we check the following simple formula:

$$
\psi(f) \psi\left(e_{n} \ldots e_{1}\right) .1=\psi(f) . e_{n} \wedge \cdots \wedge e_{1}=\prod_{i=1}^{n}\left(1+f_{i} \wedge e_{i}\right) .
$$

More generally, $\psi\left(f_{I}\right) \psi\left(e_{\bar{I}}\right) .1=\prod_{i \in I}\left(1+f_{i} \wedge e_{i}\right)$, if $\bar{I}$ denotes the sequence $I$ in reverse order.

Then we make the following series of computations, first without taking care of signs:

$$
\beta\left(e_{I}, e_{J}\right)= \pm \psi\left(e_{I}\right) \psi\left(f_{J^{c}}\right) \psi\left(f_{J}\right) \cdot e_{\bar{J}}= \pm \psi\left(e_{I}\right) \psi\left(f_{J^{c}}\right) \cdot \prod_{j \in J}\left(1+f_{j} \wedge e_{j}\right)
$$




$$
\begin{aligned}
& = \pm \psi\left(e_{I}\right) \cdot\left(f_{J^{c}} \wedge \prod_{j \in J}\left(1+f_{j} \wedge e_{j}\right)\right)= \pm \psi\left(e_{I \backslash J}\right) \psi\left(e_{I \cap J}\right) \cdot\left(f_{J^{c}} \wedge \prod_{j \in J}\left(1+f_{j} \wedge e_{j}\right)\right) \\
& = \pm 2^{|I \cap J|} \psi\left(e_{I \backslash J}\right) \cdot\left(e_{I \cap J} \wedge f_{J^{c}} \wedge \prod_{j \in J \backslash I}\left(1+f_{j} \wedge e_{j}\right)\right) \\
& = \pm 2^{|I \cap J|} \psi\left(e_{I \backslash J}\right) \cdot\left(f_{I \backslash J} \wedge f_{I^{c} \cap J^{c}} \wedge e_{I \cap J} \wedge \prod_{j \in J \backslash I}\left(1+f_{j} \wedge e_{j}\right)\right) \\
& = \pm 2^{|I \cap J|} \prod_{i \in I \backslash J}\left(1+e_{i} \wedge f_{i}\right) \wedge f_{I^{c} \cap J^{c}} \wedge e_{I \cap J} \wedge \prod_{j \in J \backslash I}\left(1+f_{j} \wedge e_{j}\right) .
\end{aligned}
$$

Note that the factor two appear because $\psi\left(e_{j}\right) \cdot\left(1+f_{j} \wedge e_{j}\right)=2 e_{j}$. Expanding the two products, one easily gets the result, up to sign.

What is the correct sign in the preceding formula? The first line contributes by the sign $\sigma\left(J^{c}, J\right)$, the signature of the permutation that puts the sequence $\left(J^{c}, J\right)$ in increasing order. Then the fourth line contributes by the sign $\sigma(I \backslash J, I \cap J)$. The sixth line contributes by the sign $\sigma\left(I \backslash J, I^{c} \cap J^{c}\right)$, and an addition minus one to the power $|I \cap J| \times\left|J^{c}\right|$ because of the permutation of the $e$ and $f$ terms. Finally, in the last lines one needs to put $I \backslash J$ in reverse order, which contributes minus one to the power $\left(\begin{array}{c}|I \backslash J| \\ 2\end{array}\right)$.

Putting things together, we obtain our quadratic equations:

Theorem 1. For any disjoint sets of integers $R, S, T$, with $R$ and $T$ of different sizes, we have

$$
\sum_{\substack{A \cup B \overline{\bar{B}}=R \cup T \\ A \cap B=\varnothing}} \epsilon(S \cup A, S \cup B, R, T) \operatorname{Pf}_{S \cup A}(u) \operatorname{Pf}_{S \cup B}(u)=0,
$$

where $\epsilon(S \cup A, S \cup B, R, T)= \pm 1$. Moreover all the quadratic relations between the sub-Pfaffians of a generic skew-symmetric matrix are linear combinations of these.

The sign function $\epsilon(S \cup A, S \cup B, R, T)$ can be written down explicitly but seemingly not in a pleasant way. These relations are probably known, but we do not know any suitable reference.

Remark 1. To be precise, the relations of the theorem will be non trivial only if the sizes of $T$ and $R$ are such that $|T|=|R|+4 p$ for some $p>0$, as follows from the properties of $\beta$. If we take this restriction into account, the theorem provides a complete set of independent quadratic relations between sub-Pfaffians.

In particular the relations found by Wenzel $[4,9]$ have to follow from these. What about the contrary? It is easy to see that Wenzel's relations are not independent. But it is quite possible that they generate the full set of quadratic relations.

\section{Decomposition formulas: cubics}

\subsection{Tensor products of fundamental representations}

The map $\beta$ allows to establish the following decomposition formulas

$$
S^{2} \Delta_{ \pm}=\wedge^{n} V_{ \pm} \oplus \bigoplus_{j>0} \wedge^{n-4 j} V, \quad \wedge^{2} \Delta_{ \pm}=\bigoplus_{j>0} \wedge^{n-4 j+2} V
$$

In the first formula $\wedge^{n} V_{+}$and $\wedge^{n} V_{-}$are the irreducible modules of highest weights $2 \omega_{n}$ and $2 \omega_{n-1}$, respectively. Also recall that $\wedge^{n-1} V$ is an irreducible but not fundamental module, its 
highest weight being $\omega_{n}+\omega_{n-1}$. This implies that it appears inside the tensor product of the two half-spin representations, which decomposes as

$$
\Delta_{+} \otimes \Delta_{-}=\wedge^{n-1} V \oplus \bigoplus_{j>0} V_{\omega_{n-2 j-1}}
$$

We aim to generalize these formulas to degree three.

Proposition 3. Let $i \leq n-2$; then

$$
\Delta_{+} \otimes \wedge^{i} V=\bigoplus_{j \geq 0} V_{\omega_{n}+\omega_{i-2 j}} \oplus \bigoplus_{j \geq 0} V_{\omega_{n-1}+\omega_{i-2 j-1}},
$$

where the first (resp. second) sum is over the set of non negative integers $j$ such that $i-2 j \geq 0$ (resp. $i-2 j+1 \geq 0)$, and we use the convention that $\omega_{0}=0$.

Proof. We first produce a non zero equivariant map from $\Delta_{+} \otimes \wedge{ }^{i} V$ to each $V_{\omega_{n}+\omega_{i-2 j}}$ or $V_{\omega_{n-1}+\omega_{i-2 j-1}}$, which will imply that the left hand side of the identity contains the right hand side. Then we prove the equality by checking dimensions.

For the first step, we observe that the action of $V$ on the spin representations induces equivariant maps $\Delta_{ \pm} \otimes \wedge^{2 j} V \rightarrow \Delta_{ \pm}$and $\Delta_{ \pm} \otimes \wedge^{2 j+1} V \rightarrow \Delta_{\mp}$. Dualizing, we obtain maps $\Delta_{ \pm} \rightarrow \Delta_{ \pm} \otimes \wedge^{2 j} V$ and $\Delta_{ \pm} \rightarrow \Delta_{\mp} \otimes \wedge^{2 j+1} V$. Hence the following sequence of equivariant morphisms,

$$
\Delta_{+} \otimes \wedge^{i} V \rightarrow \Delta_{+} \otimes \wedge^{2 j} V \otimes \wedge^{i} V \rightarrow \Delta_{+} \otimes \wedge^{i-2 j} V \rightarrow V_{\omega_{n}+\omega_{i-2 j}},
$$

where the second arrow is a contraction map by the quadratic form, and the last one is a Cartan product. Similarly, we can define the sequence

$$
\Delta_{+} \otimes \wedge^{i} V \rightarrow \Delta_{-} \otimes \wedge^{2 j+1} V \otimes \wedge^{i} V \rightarrow \Delta_{-} \otimes \wedge^{i-2 j-1} V \rightarrow V_{\omega_{n-1}+\omega_{i-2 j-1}}
$$

The composed morphisms are non zero and our first claim follows.

To check that dimensions fit, we first note that Weyl's dimension formula yields, for any $k \leq n-2$ (including $k=0$ )

$$
\operatorname{dim} V_{\omega_{n}+\omega_{k}}=\operatorname{dim} V_{\omega_{n-1}+\omega_{k}}=2^{n-1} \frac{2 n-2 k+1}{2 n-k+1}\left(\begin{array}{c}
2 n \\
k
\end{array}\right) .
$$

The required equality is thus equivalent to the identity

$$
\left(\begin{array}{c}
2 n \\
i
\end{array}\right)=\sum_{k=0}^{i} \frac{2 n-2 k+1}{2 n-k+1}\left(\begin{array}{c}
2 n \\
k
\end{array}\right)=\sum_{k=0}^{i}\left(\left(\begin{array}{c}
2 n \\
k
\end{array}\right)-\left(\begin{array}{c}
2 n \\
k-1
\end{array}\right)\right),
$$

which is obvious.

\section{Proposition 4.}

$$
\begin{aligned}
& \Delta_{+} \otimes \wedge^{n} V_{+}=V_{3 \omega_{n}} \oplus \bigoplus_{j>0} V_{\omega_{n}+\omega_{n-2 j}}, \\
& \Delta_{+} \otimes \wedge^{n} V_{-}=V_{\omega_{n}+2 \omega_{n-1}} \oplus \bigoplus_{j>0} V_{\omega_{n-1}+\omega_{n-2 j-1}} .
\end{aligned}
$$


Proof. As in the proof of the Proposition above, we first notice that there exist non-zero equivariant morphisms from the tensor products of the left hand sides of these decomposition formulas, to any irreducible component of their right hand sides.

There remains to check that dimensions fit. Weyl's dimension formula yields

$$
\operatorname{dim} V_{3 \omega_{n}}=\frac{2^{n-1}}{n+1}\left(\begin{array}{c}
2 n \\
n
\end{array}\right)=2^{n-1}\left(\left(\begin{array}{c}
2 n \\
n
\end{array}\right)-\left(\begin{array}{c}
2 n \\
n-1
\end{array}\right)\right) .
$$

The equality of dimensions is thus equivalent to the identity

$$
\frac{1}{2}\left(\begin{array}{c}
2 n \\
n
\end{array}\right)=\left(\begin{array}{c}
2 n \\
n
\end{array}\right)-\left(\begin{array}{c}
2 n \\
n-1
\end{array}\right)+\left(\begin{array}{c}
2 n \\
n-2
\end{array}\right)-\cdots+(-1)^{n}\left(\begin{array}{c}
2 n \\
0
\end{array}\right),
$$

which follows immediately from the binomial expansion of $(1-1)^{2 n}$.

In a similar way, we can prove the following identity:

\section{Proposition 5.}

$$
\Delta_{+} \otimes \wedge^{n-1} V=V_{2 \omega_{n}+\omega_{n-1}} \oplus \bigoplus_{j>0}\left(V_{\omega_{n}+\omega_{n-2 j-1}} \oplus V_{\omega_{n-1}+\omega_{n-2 j}}\right) .
$$

Of course we can deduce the corresponding identities for $\Delta_{-}$by simply exchanging $\omega_{n}$ and $\omega_{n-1}$ in the formulas above.

\subsection{Decomposing cubics}

Now we come to our main result, a decomposition formula for $S^{3} \Delta_{+}$. Since this formula will not be multiplicity free, we cannot proceed as in the preceding proofs. Instead we will use induction, and we will need some restriction formulas for certain $\mathfrak{s o}_{2 n}$-modules to $\mathfrak{s o}_{2 n-2}$. We will denote by $U$ the natural $\mathfrak{s o}_{2 n-2}$-module, the fundamental weights by $\phi_{1}, \ldots, \phi_{n-1}$, and by $U_{\phi}$ the irreducible $\mathfrak{s o}_{2 n-2}$-module with highest weight $\phi$. Also we will let $\delta_{ \pm}$be the two half-spin representations.

It is well-known that the restrictions of $\Delta_{+}$and $\Delta_{-}$are both equal to $\delta_{+} \oplus \delta_{-}$. More generally,

$$
\operatorname{Res}_{\mathfrak{s O}_{2 n-2}}^{\mathfrak{5} O_{2 n}} V_{k \omega_{n}}=\operatorname{Res}_{\mathfrak{s o}_{2 n-2}}^{\mathfrak{s o} O_{2 n}} V_{k \omega_{n-1}}=\bigoplus_{i+j=k} U_{i \phi_{n-1}+j \phi_{n-2}}
$$

To state our next results we will use the following notation: we let

$$
\begin{aligned}
& S_{i}=U_{\phi_{n-1}+\phi_{i}} \oplus U_{\phi_{n-2}+\phi_{i}} \quad \text { for } \quad 0 \leq i \leq n-3, \\
& S_{n-2}=U_{2 \phi_{n-1}+\phi_{n-2}} \oplus U_{\phi_{n-1}+2 \phi_{n-2}}, \\
& S_{n-1}=U_{3 \phi_{n-1}} \oplus U_{3 \phi_{n-2}} .
\end{aligned}
$$

Lemma 2. One has the following restriction formulas:

$$
\begin{aligned}
& \operatorname{Res}_{\mathfrak{5} 0_{2 n-2}}^{\mathfrak{5} O_{2 n}} V_{\omega_{n}+\omega_{i}}=S_{i} \oplus 2 S_{i-1} \oplus S_{i-3}, \quad \text { for } \quad 0 \leq i \leq n-2, \\
& \operatorname{Res}_{\mathfrak{s o}_{2 n-2}}^{\mathfrak{5} O_{2 n}} V_{2 \omega_{n}+\omega_{n-1}}=S_{n-1} \oplus S_{n-2} \oplus S_{n-3} .
\end{aligned}
$$

Proof. Restrict our formulas for tensor products to $\mathfrak{s o}_{2 n-2}$ and use induction.

We can now prove the main result of this section. 
Theorem 2. Let $a_{j}$, for $j \geq 0$, be the coefficients of the power series

$$
\sum_{j \geq 0} a_{j} x^{j}=\frac{1}{\left(1-x^{2}\right)\left(1-x^{3}\right)} .
$$

Then the third symmetric power of a half-spin representation decomposes as

$$
S^{3} \Delta_{+}=V_{3 \omega_{n}} \oplus \bigoplus_{j>0} a_{j} V_{\omega_{n}+\omega_{n-2 j}} \oplus \bigoplus_{j \geq 4} a_{j-4} V_{\omega_{n-1}+\omega_{n-2 j-1}}
$$

Proof. First observe that $S^{3} \Delta_{+}$is a submodule of $S^{2} \Delta_{+} \otimes \Delta_{+}$. Since the decomposition of $S^{2} \Delta_{+}$into irreducible components only involves wedge powers of the natural representation, we easily deduce from the formulas we already proved, that $S^{3} \Delta_{+}$must be a sum of modules of the form $V_{3 \omega_{n}}$ (with multiplicity one), and $V_{\omega_{n}+\omega_{n-2 j}}$ or $V_{\omega_{n-1}+\omega_{n-2 j-1}}$, with $j>0$. We can thus let

$$
S^{3} \Delta_{+}=V_{3 \omega_{n}} \oplus \bigoplus_{j>0} a_{j}^{n} V_{\omega_{n}+\omega_{n-2 j}} \oplus \bigoplus_{j>0} b_{j}^{n} V_{\omega_{n-1}+\omega_{n-2 j-1}}
$$

for some multiplicities $a_{j}^{n}, b_{j}^{n}$ which a priori, can depend on $n$. We want to compute these multiplicities inductively, by restricting this formula to $\mathfrak{s o}_{2 n-2}$. A straightforward computation yields the following relations:

$$
a_{j}^{n-1}+\alpha_{j}=2 a_{j}^{n}+b_{j}^{n}+b_{j-1}^{n}, \quad b_{j-1}^{n-1}+\beta_{j-1}=a_{j}^{n}+a_{j-1}^{n}+2 b_{j-1}^{n},
$$

where for $j \geq 1, \beta_{j}$ (resp. $\alpha_{j}$ ) is the number of pairs $(k, l)$ such that $k+2 l=j$ and $k, l \geq 0$ (resp. $k \geq 0, l \geq 1$ ). The two equations are valid for $j=1$ if we let all the coefficients with index zero equal to zero. In particular the second one yields $a_{1}^{n}=0$.

Now we can use the two equations alternatively, to compute $b_{j}^{n}$ and $a_{j}^{n}$ by induction on $j$. Indeed the first equation gives $b_{j}^{n}$ knowing $a_{j}^{n}$ (and coefficients computed before), and then the second equation gives $a_{j+1}^{n}$ knowing $a_{j}^{n}$ and $b_{j}^{n}$.

But since $\alpha_{j}$ and $\beta_{j}$ do not depend on $n$, and neither does $a_{1}^{n}$, which can be seen as the input of the induction, we can conclude that $a_{j}^{n}$ and $b_{j}^{n}$ are all independent of $n$. So we denote them simply by $a_{j}$ and $b_{j}$ and we let

$$
a(x)=\sum_{j>0} a_{j} x^{j}, \quad b(x)=\sum_{j>0} b_{j} x^{j} .
$$

Our induction relations above can then be rewritten as

$$
\begin{aligned}
& a(x)+(1+x) b(x)=\frac{x^{2}}{(1-x)\left(1-x^{2}\right)}, \\
& (1+x) a(x)+x b(x)=\frac{x}{(1-x)\left(1-x^{2}\right)}-x .
\end{aligned}
$$

Note that the determinant of the matrix of coefficients of $a(x)$ and $b(x)$ equals $(1+x)^{2}-x=$ $1+x+x^{2}$. When we solve these two equations, this explains the appearance of a factor $\left(1-x^{3}\right)$ at the denominators of $a(x)$ and $b(x)$. Indeed we easily get

$$
a(x)=\frac{1}{\left(1-x^{2}\right)\left(1-x^{3}\right)}-1, \quad b(x)=\frac{x^{4}}{\left(1-x^{2}\right)\left(1-x^{3}\right)} .
$$

The theorem is proved.

Comparing dimensions, one gets the curious corollary: 
Corollary 1. Let $c_{p}$, for $p \geq 0$, be the coefficients of the power series

$$
\sum_{p \geq 0} c_{p} x^{p}=\frac{1+x^{9}}{\left(1-x^{2}\right)\left(1-x^{3}\right)} .
$$

Then for all any integer $n \geq 2$, one has the identity

$$
\frac{\left(2^{n-1}+1\right)\left(2^{n-2}+1\right)}{3}=\sum_{p \geq 0} c_{p}\left(\left(\begin{array}{c}
2 n \\
n-p
\end{array}\right)-\left(\begin{array}{c}
2 n \\
n-p-1
\end{array}\right)\right) .
$$

Moreover, $c_{p}, p \geq 0$, is the only series with non negative coefficients satisfying this identity.

\section{Cubic equations of the secant variety}

Theorem 3. Restrictions of cubics to the secant variety of the spinor variety yields

$$
\mathbb{C}\left[\sigma\left(S_{+}\right)\right]_{3}=V_{3 \omega_{n}}^{\vee} \oplus \bigoplus_{i>1} V_{\omega_{n}+\omega_{n-2 i}}^{\vee}
$$

(Note the appearance of duals, since $S_{+} \subset \mathbb{P} \Delta_{+}$implies that $\mathbb{C}\left[\sigma\left(S_{+}\right)\right]$is a quotient of $\operatorname{Sym}\left(\Delta_{+}^{\vee}\right)$.) Comparing with the full decomposition of $S^{3} \Delta_{+}^{\vee}$ given by Theorem 2, we immediately deduce the decomposition of the space $I_{3}\left(\sigma\left(S_{+}\right)\right)$of cubic equations of the secant variety:

$$
I_{3}\left(\sigma\left(S_{+}\right)\right)=\bigoplus_{j \geq 6} a_{j-6} V_{\omega_{n}+\omega_{n-2 j}}^{\vee} \oplus \bigoplus_{j \geq 4} a_{j-4} V_{\omega_{n-1}+\omega_{n-2 j-1}}^{\vee}
$$

(Note that the term $a_{j-6}$ appears because of the relation $a(x)-\frac{x^{2}}{1-x}=x^{6}(a(x)+1)$.) In particular, we deduce:

Corollary 2. The secant variety of the spinor variety of type $D_{n}$ has non trivial cubic equations if and only if $n \geq 9$.

In type $D_{9}$ we have

$$
S^{3} \Delta_{+}=V_{3 \omega_{9}} \oplus V_{\omega_{9}+\omega_{5}} \oplus V_{\omega_{9}+\omega_{3}} \oplus V_{\omega_{9}+\omega_{1}} \oplus V_{\omega_{8}}
$$

and $I_{3}\left(\sigma\left(S_{+}\right)\right)=V_{\omega_{8}}^{\vee}$.

We can compare Theorem 3 with Theorem 3.11 in [12], according to which the coordinate ring of the tangent variety $\tau\left(S_{+}\right)$is given in degree $d$ by the formula

$$
\mathbb{C}\left[\tau\left(S_{+}\right)\right]_{d}=\bigoplus_{2 \sum a_{p} \leq \sum p a_{p} \leq d} V_{\left(d-2 \sum a_{p}\right) \omega_{n}+\sum a_{p} \omega_{n-2 p}}^{\vee},
$$

the sum being over $r$-tuples $a=\left(a_{1}, \ldots, a_{r}\right)$ of non-negative integers, with $r=\lfloor n / 2\rfloor$ (and recall the convention that $\omega_{0}=0$ ). (Actually there is a misprint in [12], where the condition stated on $a$ is not the condition that follows from the proof.) For $d=3$ we deduce the following statement:

Corollary 3. The kernel of the restriction map $\mathbb{C}\left[\sigma\left(S_{+}\right)\right]_{3} \rightarrow \mathbb{C}\left[\tau\left(S_{+}\right)\right]_{3}$ is $\bigoplus_{p>3} V_{\omega_{n}+\omega_{n-2 p}}^{\vee}$.

Proof of the theorem. We adopt the following strategy. First, we identify a set of generators of the vector spaces of equivariant maps $G-\operatorname{Hom}\left(S^{3} \Delta_{+}, V_{\omega_{n}+\omega_{n-2 i}}\right)$ and $G-\operatorname{Hom}\left(S^{3} \Delta_{+}\right.$, $\left.V_{\omega_{n-1}+\omega_{n-2 i-1}}\right)$, for each $i$. Then we evaluate these generators at a general point of the secant variety and prove that their image has rank one and zero, respectively. 
First step. By Theorem 2, we know that the dimension of $G-\operatorname{Hom}\left(S^{3} \Delta_{+}, V_{\omega_{n}+\omega_{n-2 i}}\right)$ is equal to the number of pairs $(a, b)$ of integers such that $2 a+3 b=i$. Rather than constructing an explicit basis, it will be much easier to construct one of $G-\operatorname{Hom}\left(S^{2} \Delta_{+} \otimes \Delta_{+}, V_{\omega_{n}+\omega_{n-2 i}}\right)$. By restriction to $S^{3} \Delta_{+} \subset S^{2} \Delta_{+} \otimes \Delta_{+}$, we will deduce a set of generators of $G-\operatorname{Hom}\left(S^{3} \Delta_{+}, V_{\omega_{n}+\omega_{n-2 i}}\right)$. Recall the decomposition

$$
S^{2} \Delta_{+}=\wedge^{n} V_{+} \oplus \bigoplus_{j>0} \wedge^{n-4 j} V
$$

By Proposition 3, there exists an equivariant map from $\wedge^{n-4 j} V \otimes \Delta_{+}$to $V_{\omega_{n}+\omega_{n-2 i}}$ if and only if $i \geq 2 j$. Moreover this map is then unique (up to constant), and can be described as the following composition:

$$
\phi_{i, j}: \quad \wedge^{n-4 j} V \otimes \Delta_{+} \longrightarrow \wedge^{n-2 i} V \otimes \wedge^{2 i-4 j} V \otimes \Delta_{+} \longrightarrow \wedge^{n-2 i} V \otimes \Delta_{+} \longrightarrow V_{\omega_{n}+\omega_{n-2 i}} .
$$

Here the first arrow is induced by the dual map to the exterior multiplication of exterior forms; the second one by the action of the Clifford algebra (which is isomorphic to the exterior algebra) on the half-spin representations; the last one is the projection to the Cartan component.

The $\phi_{i, j}$, where $i \geq 2 j$, form a basis of $G-\operatorname{Hom}\left(S^{2} \Delta_{+} \otimes \Delta_{+}, V_{\omega_{n}+\omega_{n-2 i}}\right)$.

Second step. Now we need to evaluate $\phi_{i, j}$ on a generic point of the secant variety, which will of course be our favourite point introduced at the very end of Section 2.2.

We will consider the case were $n=2 m$ is even, the case $n$ odd being similar. Then $u_{E}=$ $e_{1} \cdots e_{n}=e_{\max }$ and $u_{F}=1=e_{\varnothing}$. We first evaluate the image $\psi_{2 i, 2 j}$ of $e_{\max } e_{\varnothing} \otimes e_{\varnothing}$ inside $\wedge^{n-2 i} V \otimes \Delta_{+}$.

Lemma 3. Up to a non zero constant,

$$
\psi_{2 i, 2 j}=\sum_{\substack{\ell(I)=m-i-j \\ \ell(J)=2 j}} e_{I} \wedge f_{I} \wedge f_{J} \otimes e_{J} .
$$

What remains to do is to evaluate the dimension of the subspace of $V_{\omega_{n}+\omega_{n-2 i}}$ spanned by the images $\bar{\psi}_{2 i, 2 j}$ of the $\psi_{2 i, 2 j}, i \geq 2 j$. It turns out that this dimension is equal to one, because of the following dependence relations.

Lemma 4. For $j \geq 1$ and $i+j \leq m$, we have

$$
(-1)^{m-i-j}(2 j-1) \bar{\psi}_{2 i, 2 j}+(m+i-j+1) \bar{\psi}_{2 i, 2 j-2}=0 \text {. }
$$

Proof. First observe that the natural maps $V \otimes \Delta_{ \pm} \rightarrow \Delta_{\mp}$ induce, by transposition, maps $\Delta_{ \pm} \rightarrow \Delta_{\mp} \otimes V^{\vee} \simeq \Delta_{\mp} \otimes V$. We can thus define an equivariant morphism

$$
\kappa_{i}: \wedge^{n-2 i-1} V \otimes \Delta_{-} \longrightarrow \wedge^{n-2 i-1} V \otimes V \otimes \Delta_{+} \longrightarrow \wedge^{n-2 i} V \otimes \Delta_{+} .
$$

Observe that the image of this map is contained in (and actually coincides with, but we will not need that) the kernel of the projection to the Cartan component $V_{\omega_{n}+\omega_{n-2 i}}$. Indeed, it follows from Proposition 3 that the tensor product $\wedge^{n-2 i-1} V \otimes \Delta_{-}$does not contain any copy of $V_{\omega_{n}+\omega_{n-2 i}}$.

Now consider $\psi_{2 i+1,2 j-1} \in \wedge^{n-2 i-1} V \otimes \Delta_{-}$. Its image by $\kappa_{i}$ is

$$
\kappa_{i}\left(\psi_{2 i+1,2 j-1}\right)=\sum_{k=1}^{n}\left(\sum_{\substack{\ell(I)=m-i-j \\ \ell(J)=2 j-1}} e_{k} \wedge e_{I} \wedge f_{I} \wedge f_{J} \otimes f_{k} . e_{J}+f_{k} \wedge e_{I} \wedge f_{I} \wedge f_{J} \otimes e_{k} \wedge e_{J}\right)
$$




$$
\begin{aligned}
& =\sum_{k=1}^{n}\left(\sum_{k \in J}(-1)^{\ell(I)} e_{I \cup k} \wedge f_{I \cup k} \wedge f_{J \backslash k} \otimes e_{J \backslash k}+\sum_{k \notin I \cup J} e_{I} \wedge f_{I} \wedge f_{J \cup k} \otimes e_{J \cup k}\right) \\
& =(-1)^{m-i-j}(2 j-1) \psi_{2 i, 2 j-2}+(m+i-j+1) \psi_{2 i, 2 j} .
\end{aligned}
$$

As we have seen the projection of $\kappa_{i}\left(\psi_{2 i+1,2 j-1}\right)$ in $V_{\omega_{n}+\omega_{n-2 i}}$ must be zero, and this implies the claim.

The coefficients in the previous dependence relations are always non zero, so the dimension of the span of the $\bar{\psi}_{2 i, 2 j}$ is at most one. What remains to check is that it is non zero, which follows from the next lemma.

Lemma 5. $\bar{\psi}_{2 i, 0} \neq 0$.

Proof. Recall that $\psi_{2 i, 0}=\sum_{\ell(I)=m-i} e_{I} \wedge f_{I} \otimes 1 \in \wedge^{n-2 i} V \otimes \Delta_{+}$. We need to prove that this tensor has a non zero Cartan component. To detect the component on $V_{\omega_{n}+\omega_{n-2 i}}$, we just need to pair $\psi_{2 i, 0}$ with a highest weight vector in this representation, that is, a tensor of the form $g_{1} \wedge \cdots \wedge g_{n-2 i} \otimes u_{G}$, where $G$ is a maximal isotropic space in the same family as $E$, and the vectors $g_{1}, \ldots, g_{n-2 i}$ belong to $G$. Generically, the pairing of such a tensor with $\psi_{2 i, 0}$ is clearly non zero: this is obvious for the pairing of 1 with $u_{G}$ in $\Delta_{+}$, which is the top-degree component of $u_{G}$; and also for the pairing with $g_{1} \wedge \cdots \wedge g_{n-2 i}$, since such tensors generate the full $\wedge^{n-2 i} V$.

Now we consider cubics of type $V_{\omega_{n-1}+\omega_{n-2 i-1}}$. As before we first identify a basis of $G-$ $\operatorname{Hom}\left(S^{2} \Delta_{+} \otimes \Delta_{+}, V_{\omega_{n-1}+\omega_{n-2 i-1}}\right)$, given by the composition $\phi_{i, j}^{-}$of the following natural maps

$$
\begin{aligned}
\phi_{i, j}^{-}: \quad \wedge^{n-4 j} V \otimes \Delta_{+} & \longrightarrow \wedge^{n-2 i-1} V \otimes \wedge^{2 i-4 j+1} V \otimes \Delta_{+} \\
& \longrightarrow \wedge^{n-2 i-1} V \otimes \Delta_{-} \longrightarrow V_{\omega_{n-1}+\omega_{n-2 i-1}} .
\end{aligned}
$$

Again for $n=2 m$ even, we evaluate $\phi_{i, j}^{-}$at the same point as before, and we get, up to a non zero constant, the tensor $\bar{\psi}_{2 i+1,2 j+1}$. As before, when $j$ varies, the $\bar{\psi}_{2 i+1,2 j+1}$ are modified only by a non zero constant. And this implies that they are all equal to zero, because

Lemma 6. $\bar{\psi}_{2 i+1,1}=0$.

Proof. Indeed, the very same computation as that of Lemma 4 shows that $\psi_{2 i+1,1}$ is a non zero multiple of $\kappa_{i}\left(\psi_{2 i, 0}\right)$, and therefore its projection to $V_{\omega_{n-1}+\omega_{n-2 i-1}}$ has to vanish.

\section{Decomposition formulas: quartics}

\subsection{More formulas for tensor products}

First we shall need decomposition formulas for tensor products of some Cartan powers of spin representations. We use the notation $\theta_{i}=\epsilon_{1}+\cdots+\epsilon_{i}$; this is a fundamental weight $\omega_{i}$ when $i \leq n-2$, but $\theta_{n-1}=\omega_{n-1}+\omega_{n}$ and $\theta_{n}=2 \omega_{n}$. We have

$$
\begin{array}{ll}
V_{3 \omega_{n}} \otimes \Delta_{+}=\bigoplus_{j \geq 0} V_{\theta_{n}+\theta_{n-2 j}}, & V_{3 \omega_{n}} \otimes \Delta_{-}=\bigoplus_{j \geq 0} V_{\theta_{n}+\theta_{n-2 j-1}}, \\
V_{2 \omega_{n}} \otimes V_{2 \omega_{n-1}}=\bigoplus_{j, k \text { odd }} V_{\theta_{n-j}+\theta_{n-k}}, & V_{2 \omega_{n}} \otimes V_{\omega_{n-i}}=\bigoplus_{\substack{j+k \geq i \\
j+k-i \text { even }}} V_{\theta_{n-j}+\theta_{n-k}},
\end{array}
$$


Observe that, as follows from Weyl's dimension formula, for $p \geq q \geq 1$,

$$
\begin{aligned}
& \operatorname{dim} V_{\theta_{n}+\theta_{n-p}}=(p+1)^{2} \frac{(2 n) !(2 n+1) !}{(n-p) !(n+p+2) ! n !(n+1) !}, \\
& \operatorname{dim} V_{\theta_{n-p}+\theta_{n-q}}=(p-q+1)(p+q+1) \frac{(2 n) !(2 n+2) !}{(n-p) !(n+p+2) !(n-q+1) !(n+q+1) !} .
\end{aligned}
$$

We will also need formulas for the tensor products of fundamental non spin representations. These are just wedge products of the vector representations, so one can take their tensor products as $\mathfrak{s l}_{2 n}$-modules, and then restrict to $\mathfrak{s} O_{2 n}$-modules using the Littlewood restriction rules and their generalization by King and Howe-Tan-Willenbring (see [5]). The result is the following decomposition formula, for $p \geq q$ :

$$
V_{\omega_{n-p}} \otimes V_{\omega_{n-q}}=\bigoplus_{\substack{p-q \leq r-s \leq p+q \leq r+s \\
p+q-r-s \text { even }}} V_{\theta_{n-r}+\theta_{n-s}} \oplus 2 \bigoplus_{\begin{array}{c}
p+q \leq r+s \\
p+q-r-s \text { even }
\end{array}} V_{\theta_{n-r}+\theta_{n-s}} .
$$

For future use we will need to understand in some detail the spin-equivariant maps $V_{\omega_{p}} \otimes V_{\omega_{q}} \rightarrow$ $V_{\theta_{r}+\theta_{s}}$. Observe that we can define two basic maps

$$
\begin{aligned}
& \alpha_{p, q}^{p-1, q+1}: \Lambda^{p} V \otimes \Lambda^{q} V \rightarrow \Lambda^{p-1} V \otimes V \otimes \Lambda^{q} V \rightarrow \Lambda^{p-1} V \otimes \Lambda^{q+1} V, \\
& \alpha_{p, q}^{p-1, q-1}: \Lambda^{p} V \otimes \Lambda^{q} V \rightarrow \Lambda^{p-1} V \otimes V \otimes \Lambda^{q} V \rightarrow \Lambda^{p-1} V \otimes \Lambda^{q-1} V .
\end{aligned}
$$

In the definition of $\alpha_{p, q}^{p-1, q+1}$ we used the natural map $V \otimes \Lambda^{q} V \rightarrow \Lambda^{q+1} V$ defined by the wedge product, while in the definition of $\alpha_{p, q}^{p-1, q-1}$ we used the map $V \otimes \Lambda^{q} V \rightarrow \Lambda^{q-1} V$ induced by the contraction by the quadratic form on $V$.

A straightforward computation shows that

$$
\alpha_{p-1, q+1}^{p-2, q} \circ \alpha_{p, q}^{p-1, q+1}=\alpha_{p-1, q-1}^{p-2, q} \circ \alpha_{p, q}^{p-1, q-1} .
$$

By such compositions, we can therefore define unambiguously, maps

$$
\alpha_{p, q}^{r, s}: \Lambda^{p} V \otimes \Lambda^{q} V \rightarrow \Lambda^{r} V \otimes \Lambda^{s} V
$$

for $p+q-r-s$ even and $|q-s| \leq p-r$. Note that the latter condition is equivalent to $p-q \geq r-s$ and $p+q \geq r+s$.

$\Lambda^{p} V \otimes \Lambda^{q} V$

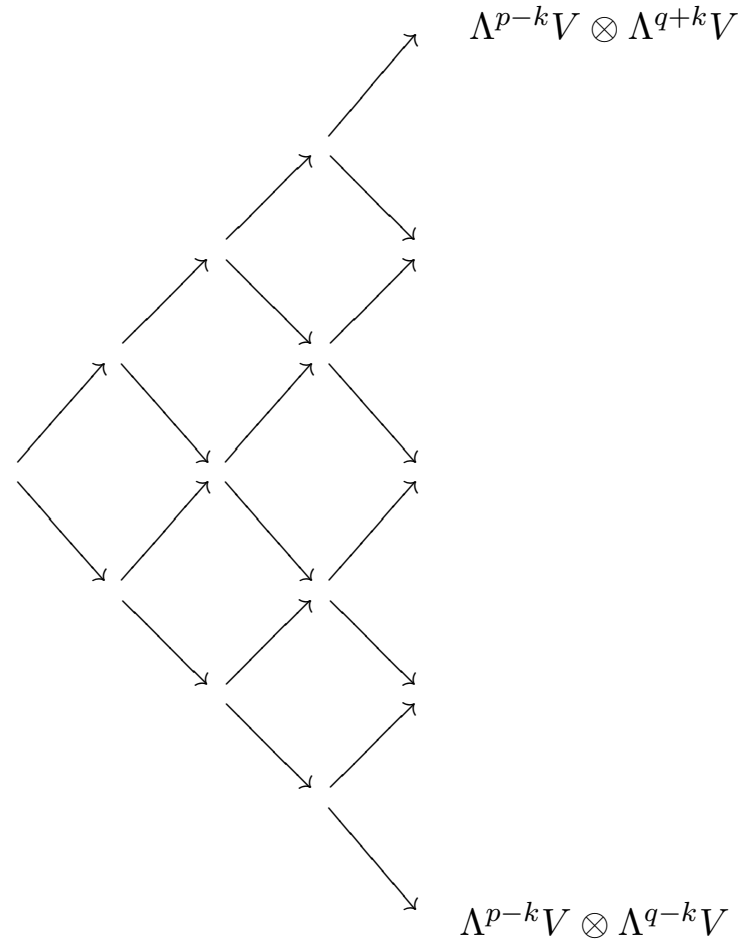


If we compose such a map $\alpha_{p, q}^{r, s}$ with the projection to the Cartan component,

$$
\Lambda^{p} V \otimes \Lambda^{q} V \rightarrow \Lambda^{r} V \otimes \Lambda^{s} V \rightarrow V_{\theta_{r}+\theta_{s}}
$$

we claim that the resulting map $\beta_{p, q}^{r, s}$ is non zero. This is also true if $s \geq n$, which can occur if $p+q \geq n$; in this case, since $\Lambda^{s} V \simeq \Lambda^{2 n-s} V$, the resulting map $\beta_{p, q}^{r, s}$ maps $\Lambda^{p} V \otimes \Lambda^{q} V$ to $V_{\theta_{r}+\theta_{2 n-s}}$. We claim that $\beta_{p, q}^{r, s}$ and $\beta_{p, q}^{r, 2 n-s}$ are independent, and that this is the explanation for the multiplicities that are equal to two in the decomposition formula above.

\subsection{Branching}

Second, we will use restriction formulas for representations of type $V_{\theta_{i}+\theta_{j}}$, first from $D_{n+1}$ to $B_{n}$, then to $D_{n}$ (see again [5]). We identify the weight $\theta_{i}+\theta_{j}$, for $i \leq j$, with the partition $\left(2^{i} 1^{j-i}\right)$, of length $j \leq n+1$.

1. To obtain the restriction of $V_{\theta_{i}+\theta_{j}}$ from $D_{n+1}$ to $B_{n}$, take the sum of the representations defined by the partitions

$$
\left(2^{i} 1^{j-i}\right), \quad\left(2^{i-1} 1^{j-i+1}\right), \quad\left(2^{i} 1^{j-i-1}\right), \quad\left(2^{i-1} 1^{j-i}\right),
$$

(where only those of length at most $n$ must be kept).

2. To obtain the restriction of $V_{\theta_{i}+\theta_{j}}$ from $B_{n}$ to $D_{n}$, make the same operation, except that if one of the representation obtained is of the form $V_{\theta_{n}+\theta_{i}}=V_{2 \omega_{n}+\theta_{i}}\left(\right.$ resp. $\left.V_{2 \theta_{n}}=V_{4 \omega_{n}}\right)$, one has to add the mirror representation $V_{2 \omega_{n-1}+\theta_{i}}$ (resp. $\left.V_{4 \omega_{n-1}}\right)$.

\subsection{Decomposing quartics}

From the preceding formulas, and our decomposition for cubics, we easily deduce the following statement:

Proposition 6. There exists integers $e_{i, j}^{n}$ and $f_{i}^{n}$ such that the decomposition formula for the fourth symmetric power of a half-spin representation is of the form

$$
S^{4} \Delta_{+}=\bigoplus_{0 \leq i, j \leq n} e_{i, j}^{n} V_{\theta_{n-i}+\theta_{n-j}} \oplus \bigoplus_{0 \leq i \leq n} f_{i}^{n} V_{2 \omega_{n-1}+\theta_{n-i}} .
$$

Moreover $e_{i, j}^{n}=0$ if $i+j$ is odd and $f_{i}^{n}=0$ if $i$ is odd.

Proof. Only the last assertion needs to be proved. Recall that the weights of the half-spin representation, after a suitable choice of a maximal torus of $\operatorname{Spin}(V)$, are of the form $\frac{1}{2}\left( \pm \epsilon_{1} \pm\right.$ $\cdots \pm \epsilon_{n}$ ), where the number of minus signs is even. In particular the sum of the coefficients is $\frac{n}{2}$ minus some even integer. This implies that if $a_{1} \epsilon_{1}+\cdots+a_{n} \epsilon_{n}$ is any weight of $S^{4} \Delta_{+}$, then $a_{1}+\cdots+a_{n}$ equals $2 n \bmod 2$. In particular, if $\theta_{n-i}+\theta_{n-j}$ is a weight of $S^{4} \Delta_{+}$, then $i+j$ must be even. Similarly, if $2 \omega_{n-1}+\theta_{n-i}$ is a weight of $S^{4} \Delta_{+}$, then $i$ must be even.

We expect the same phenomena as for cubics, that is:

Conjecture. The integers $e_{i, j}^{n}$ and $f_{i}^{n}$ are independent of $n$.

We have checked this conjecture up to $n=20$ with the help of the program LiE [14].

The coefficients $f_{i}=f_{i}^{n}$ are all equal to zero in that range, except $f_{16}=f_{20}=1$. The first coefficients $e_{i, j}=e_{i, j}^{n}$ are given by the following table, where they are displayed in such a way 
that the first line gives the coefficients $e_{0, j}$, and the diagonal gives the coefficients $e_{i, i}$.

$$
\begin{aligned}
& \begin{array}{lllllllllllllllllllll}
1 & 0 & 0 & 0 & 1 & 0 & 1 & 0 & 2 & 0 & 1 & 0 & 3 & 0 & 2 & 0 & 4 & 0 & 3 & 0 & 5
\end{array} \\
& \begin{array}{llllllllllllllllllll}
0 & 0 & 0 & 0 & 0 & 0 & 0 & 0 & 1 & 0 & 1 & 0 & 2 & 0 & 2 & 0 & 3 & 0 & 4 & 0
\end{array} \\
& \begin{array}{lllllllllllllllllll}
0 & 0 & 0 & 0 & 1 & 0 & 0 & 0 & 2 & 0 & 1 & 0 & 3 & 0 & 2 & 0 & 5 & 0 & 3
\end{array} \\
& \begin{array}{llllllllllllllllll}
0 & 0 & 0 & 0 & 1 & 0 & 1 & 0 & 1 & 0 & 2 & 0 & 3 & 0 & 3 & 0 & 4 & 0
\end{array} \\
& \begin{array}{lllllllllllllllll}
1 & 0 & 0 & 0 & 1 & 0 & 1 & 0 & 3 & 0 & 1 & 0 & 4 & 0 & 3 & 0 & 6
\end{array} \\
& \begin{array}{llllllllllllllll}
0 & 0 & 0 & 0 & 1 & 0 & 0 & 0 & 2 & 0 & 2 & 0 & 3 & 0 & 3 & 0
\end{array}
\end{aligned}
$$

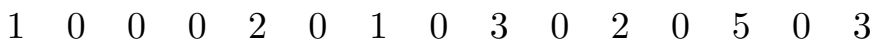

$$
\begin{aligned}
& 0 \begin{array}{llllllllllllll}
0 & 0 & 0 & 0 & 1 & 0 & 1 & 0 & 2 & 0 & 2 & 0 & 4 & 0
\end{array} \\
& \begin{array}{lllllllllllll}
1 & 0 & 0 & 0 & 2 & 0 & 1 & 0 & 4 & 0 & 2 & 0 & 5
\end{array} \\
& \begin{array}{llllllllllll}
1 & 0 & 0 & 0 & 2 & 0 & 1 & 0 & 3 & 0 & 3 & 0
\end{array} \\
& \begin{array}{lllllllllll}
1 & 0 & 0 & 0 & 2 & 0 & 1 & 0 & 4 & 0 & 2
\end{array} \\
& \begin{array}{llllllllll}
0 & 0 & 0 & 0 & 2 & 0 & 1 & 0 & 3 & 0
\end{array} \\
& \begin{array}{lllllllll}
2 & 0 & 0 & 0 & 3 & 0 & 2 & 0 & 5
\end{array} \\
& \begin{array}{llllllll}
1 & 0 & 0 & 0 & 2 & 0 & 1 & 0
\end{array} \\
& \begin{array}{lllllll}
1 & 0 & 0 & 0 & 3 & 0 & 1
\end{array} \\
& \begin{array}{llllll}
1 & 0 & 0 & 0 & 3 & 0
\end{array} \\
& \begin{array}{lllll}
2 & 0 & 0 & 0 & 3
\end{array} \\
& \begin{array}{llll}
1 & 0 & 0 & 0
\end{array} \\
& \begin{array}{lll}
2 & 0 & 0
\end{array} \\
& 10
\end{aligned}
$$

We can try to prove this conjecture in the same way as we proved the similar statement for cubics, that is, by induction on $n$. So we restrict the above formula for $S^{4} \Delta_{+}$to $D_{n-1}$ and deduce an inductive relation for the multiplicities.

To obtain this equation we need a formula for the tensor products of $S^{3} \Delta_{+} \otimes \Delta_{-}$. One can check that for $p \geq 2$,

$$
V_{\omega_{n}+\omega_{n-p}} \otimes \Delta_{+}=\bigoplus_{\substack{r \geq p \geq s \\ r+s-p \text { even }}} V_{\theta_{n-r}+\theta_{n-s}}, \quad V_{\omega_{n}+\omega_{n-p}} \otimes \Delta_{-}=\bigoplus_{\substack{r \geq p \geq s \\ r+s-p \text { odd }}} V_{\theta_{n-r}+\theta_{n-s}}
$$

One can then write down some inductive relations for the multiplicities $e_{i, j}^{n}$ and $f_{i}^{n}$, which unfortunately are not sufficient to compute them all (contrary to the cubic case), and a fortiori not sufficient to prove the conjecture: some additional idea is needed.

Admitting the conjecture, we obtain the following recursive relations for the multiplicities:

$$
\begin{aligned}
& e_{i-1, i-1}+e_{i, i}+e_{i+1, i+1}=\left\lfloor\frac{i}{4}\right\rfloor+\delta_{i, 0}+\delta_{i, o d d}, \\
& e_{0,2 i}^{\prime}+e_{1,2 i-1}+e_{1,2 i+1}=\left(\begin{array}{c}
\left\lfloor\frac{i}{2}\right\rfloor+1 \\
2
\end{array}\right), \\
& e_{i-1, j}+e_{i, j-1}+e_{i+1, j}+e_{i, j+1}=\sum_{i \leq 2 p \leq j} a_{p}+\sum_{i \leq 2 p+1 \leq j} b_{p},
\end{aligned}
$$

where we have let $e_{0, j}^{\prime}=e_{0, j}+f_{j}$. Moreover the third type of equations holds for $j>i>0$, with the caveat that for $i=1, e_{0, j}$ has to be replaced by $e_{0, j}^{\prime}$.

The first series of equations allow to compute the diagonal coefficients $e_{i, i}$. Their generating series is

$$
\sum_{i \geq 0} e_{i, i} x^{i}=\frac{1+x^{9}}{\left(1-x^{4}\right)\left(1-x^{6}\right)}
$$


We conjecture that the generating series of the multiplicities $e_{0, i}$ and $f_{i}$ are

$$
\sum_{i \geq 0} e_{0, i} x^{i}=\frac{1}{\left(1-x^{4}\right)\left(1-x^{6}\right)\left(1-x^{8}\right)}, \quad \sum_{i \geq 0} f_{i} x^{i}=\frac{x^{16}}{\left(1-x^{4}\right)\left(1-x^{6}\right)\left(1-x^{8}\right)} .
$$

Once the coefficients $e_{i, i}, e_{0, i}$ and $f_{i}$ are known, the recursive relations above allow to compute all the multiplicities $e_{i, j}$, and to write down their generating series as an explicit rational function.

\section{Quartic equations of the secant variety}

We first make the following easy observation (see [11]): the space $I_{4}\left(\sigma\left(S_{+}\right)\right)$of quartic equations of the secant variety to the spinor variety, is the orthogonal in $S^{4} \Delta_{+}^{\vee}$ to the subspace of $S^{4} \Delta_{+}$ generated by the fourth powers $(a+b)^{4}$, where $a, b$ are pure spinors (i.e., belong to the cone over $\left.S_{+}\right)$. Such a tensor decomposes into homogeneous components that can be treated separately: the fourth powers $a^{4}$ generate the Cartan components $V_{4 \omega_{n}}$ of $S^{4} \Delta_{+}$; the terms $a^{3} b$ generate the image of $V_{3 \omega_{n}} \otimes V_{\omega_{n}} \subset S^{3} \Delta_{+} \otimes \Delta_{+}$in $S^{4} \Delta_{+}$; the terms $a^{2} b^{2}$ generate the image of $V_{2 \omega_{n}} \otimes V_{2 \omega_{n}}$ (or rather its symmetric part). These tensor products are known: we have

$$
V_{3 \omega_{n}} \otimes V_{\omega_{n}}=\bigoplus_{p} V_{\theta_{n}+\theta_{n-2 p}}, \quad S^{2} V_{2 \omega_{n}}=\bigoplus_{p-q \text { even }} V_{\theta_{n-2 p}+\theta_{n-2 q}}
$$

The only components that appear in both decomposition are $V_{4 \omega_{n}}$ and the $V_{\theta_{n}+\theta_{n-2 p}}$ 's, for $p$ an even integer. This implies that

$$
\mathbb{C}\left[\sigma\left(S_{+}\right)\right]_{4} \subset \bigoplus_{\substack{p \geq q>0 \\ p-q \text { even }}} V_{\theta_{n-2 p}+\theta_{n-2 q}}^{\vee} \oplus 2 \bigoplus_{p \text { even }} V_{\theta_{n}+\theta_{n-2 p}}^{\vee} \oplus \bigoplus_{p \text { odd }} V_{\theta_{n}+\theta_{n-2 p}}^{\vee} .
$$

\section{Theorem 4.}

$$
\mathbb{C}\left[\sigma\left(S_{+}\right)\right]_{4}=\bigoplus_{\substack{p \geq q \geq 1 \\ p-q \text { even } \\(p, q) \neq(1,1)}} V_{\theta_{n-2 p}+\theta_{n-2 q}}^{\vee} \oplus \bigoplus_{p \neq 1} V_{\theta_{n}+\theta_{n-2 p}}^{\vee}
$$

Proof. There are two things to prove. First, that the $V_{\theta_{n}+\theta_{n-2 p}}^{\vee}$ 's, for $p$ even, appear in $\mathbb{C}\left[\sigma\left(S_{+}\right)\right]_{4}$ with multiplicity one. Second, that the $V_{\theta_{n-2 p}+\theta_{n-2 q}}^{\vee}$ 's, for $p-q$ even, or $q=0$ and $p$ odd, have non zero multiplicity.

For the first assertion, recall that $V_{\theta_{n-2 p}}$ is, for $p$ even, a component of $S^{2} \Delta_{+}$, with multiplicity one. Let $V=\sum_{i} v_{i} \otimes v_{i}^{\prime}$ be a generator of the corresponding highest weight line in $S^{2} \Delta_{+} \subset$ $\Delta_{+} \otimes \Delta_{+}$. Denote by $u$ a highest weight vector of $\Delta_{+}$. Then $\sum_{i}\left(u v_{i}\right) \otimes\left(u v_{i}^{\prime}\right)$ is a highest weight vector in $S^{2} V_{2 \omega_{n}}$, while $\sum_{i}\left(u^{2} v_{i}\right) \otimes v_{i}^{\prime}$ is a highest weight vector in $V_{3 \omega_{n}} \otimes V_{\omega_{n}}$. Since their images in $S^{4} V_{2 \omega_{n}}$ are both equal to $\sum_{i} u^{2} v_{i} v_{i}^{\prime}$, we deduce that the components $V_{\theta_{n}+\theta_{n-2 p}}$ inside $S^{2} V_{2 \omega_{n}}$ and inside $V_{3 \omega_{n}} \otimes V_{\omega_{n}}$ generate a unique copy of $V_{\theta_{n}+\theta_{n-2 p}}$ inside $S^{4} V_{2 \omega_{n}}$. This proves the first claim.

For the second assertion, consider a component $V_{\theta_{n-2 p}+\theta_{n-2 q}}$ coming from $S^{2} V_{2 \omega_{n}}$, with $p \geq q$. We want to check that the tensors of the form $e^{2} f^{2}$ in $S^{4} V_{2 \omega_{n}}$, for $e$ and $f$ in the cone over the spinor variety, do generate such a component.

Suppose first that $p$ and $q$ are both even. Consider the map

$$
S^{2} V_{2 \omega_{n}} \hookrightarrow S^{2} V_{\omega_{n}} \otimes S^{2} V_{\omega_{n}} \stackrel{\beta_{n-2 p} \otimes \beta_{n-2 q}}{\longrightarrow} V_{\theta_{n-2 p}} \otimes V_{\theta_{n-2 q}} .
$$

Since $\beta_{n-2 p}(e, e)=0$ (at least for $p \geq 1$ ), the tensor $e^{2} f^{2}$ is mapped by this morphism to

$$
\beta_{n-2 p}(e, f) \otimes \beta_{n-2 q}(e, f) \in V_{\theta_{n-2 p}} \otimes V_{\theta_{n-2 q}} .
$$


To check that $\beta_{n-2 p}(e, f) \otimes \beta_{n-2 q}(e, f)$ has a non zero projection to the Cartan component $V_{\theta_{n-2 p}+\theta_{n-2 q}}$, we just need to pair it with highest weight vectors of that component (which is self-dual). Such highest weight vectors are of the form

$$
g_{1} \wedge \cdots \wedge g_{n-2 p} \otimes g_{1} \wedge \cdots \wedge g_{n-2 q},
$$

where the vectors $g_{1}, \ldots, g_{n-2 q}$ generate an isotropic subspace of $V$ (recall that we have supposed $p \geq q)$. So it suffices to check that $\beta_{n-2 p}(e, f)$ pairs non trivially with a generic tensor $g_{1} \wedge \cdots \wedge$ $g_{n-2 p}$. But this is obvious, since such tensors generate the whole space $V_{\theta_{n-2 p}}$.

Now suppose that $p$ and $q$ are both odd. Then we cannot use the same morphism as before, and instead we use the composition

$$
S^{2} V_{2 \omega_{n}} \hookrightarrow S^{2} V_{\omega_{n}} \otimes S^{2} V_{\omega_{n}} \stackrel{\beta_{n-2 p+2} \otimes \beta_{n-2 q-2}}{\longrightarrow} V_{\theta_{n-2 p+2}} \otimes V_{\theta_{n-2 q-2}} \longrightarrow V_{\theta_{n-2 p}} \otimes V_{\theta_{n-2 q}}
$$

We conclude as in the previous case, except when $p=1$ for which we get zero. By symmetry we can always get the component $V_{\theta_{n-2 p}+\theta_{n-2 q}}$, except in the case where $(p, q)=(1,1)$.

Comparing with the decomposition of $S^{4} \Delta_{+}^{\vee}$ we can of course deduce the decomposition of $I_{4}\left(\sigma\left(S_{+}\right)\right)$into irreducible components. It has the same "odd" part (by this we mean the same multiplicities on the components of type $V_{2 \omega_{n-1}+\theta_{n-j}}$ ), while the multiplicities of components $V_{\theta_{n-i}+\theta_{n-j}}$ in the "even" part are given, for low values of $i$ and $j$, by the following table:

$$
\begin{aligned}
& \begin{array}{lllllllllllllllllllll}
0 & 0 & 0 & 0 & 0 & 0 & 0 & 0 & 1 & 0 & 0 & 0 & 2 & 0 & 1 & 0 & 3 & 0 & 2 & 0 & 4
\end{array} \\
& \begin{array}{llllllllllllllllllll}
0 & 0 & 0 & 0 & 0 & 0 & 0 & 0 & 1 & 0 & 1 & 0 & 2 & 0 & 2 & 0 & 3 & 0 & 4 & 0
\end{array} \\
& \begin{array}{lllllllllllllllllll}
0 & 0 & 0 & 0 & 0 & 0 & 0 & 0 & 1 & 0 & 1 & 0 & 2 & 0 & 2 & 0 & 4 & 0 & 3
\end{array} \\
& \begin{array}{llllllllllllllllll}
0 & 0 & 0 & 0 & 1 & 0 & 1 & 0 & 1 & 0 & 2 & 0 & 3 & 0 & 3 & 0 & 4 & 0
\end{array} \\
& \begin{array}{lllllllllllllllll}
0 & 0 & 0 & 0 & 0 & 0 & 1 & 0 & 2 & 0 & 1 & 0 & 3 & 0 & 3 & 0 & 5
\end{array} \\
& \begin{array}{llllllllllllllll}
0 & 0 & 0 & 0 & 1 & 0 & 0 & 0 & 2 & 0 & 2 & 0 & 3 & 0 & 3 & 0
\end{array} \\
& 0 \begin{array}{lllllllllllllll}
0 & 0 & 0 & 0 & 1 & 0 & 1 & 0 & 2 & 0 & 2 & 0 & 4 & 0 & 3
\end{array} \\
& \begin{array}{llllllllllllll}
0 & 0 & 0 & 0 & 1 & 0 & 1 & 0 & 2 & 0 & 2 & 0 & 4 & 0
\end{array} \\
& \begin{array}{lllllllllllll}
0 & 0 & 0 & 0 & 1 & 0 & 1 & 0 & 3 & 0 & 2 & 0 & 4
\end{array} \\
& \begin{array}{llllllllllll}
1 & 0 & 0 & 0 & 2 & 0 & 1 & 0 & 3 & 0 & 3 & 0
\end{array} \\
& \begin{array}{lllllllllll}
0 & 0 & 0 & 0 & 1 & 0 & 1 & 0 & 3 & 0 & 2
\end{array} \\
& \begin{array}{llllllllll}
0 & 0 & 0 & 0 & 2 & 0 & 1 & 0 & 3 & 0
\end{array} \\
& \begin{array}{lllllllll}
1 & 0 & 0 & 0 & 2 & 0 & 2 & 0 & 4
\end{array} \\
& \begin{array}{llllllll}
1 & 0 & 0 & 0 & 2 & 0 & 1 & 0
\end{array} \\
& \begin{array}{lllllll}
0 & 0 & 0 & 0 & 2 & 0 & 1
\end{array} \\
& \begin{array}{llllll}
1 & 0 & 0 & 0 & 3 & 0
\end{array} \\
& \begin{array}{lllll}
1 & 0 & 0 & 0 & 2
\end{array} \\
& \begin{array}{llll}
1 & 0 & 0 & 0
\end{array} \\
& \begin{array}{lll}
1 & 0 & 0
\end{array} \\
& \begin{array}{ll}
1 & 0
\end{array} \\
& 1
\end{aligned}
$$

Of course these multiplicities will be independent of $n$ if and only if the conjecture in Section 5.3 is correct.

For example, we get (denoting $S_{n}$ the spinor variety $S_{+}$in type $D_{n}$ ):

$$
\begin{aligned}
& I_{4}\left(\sigma\left(S_{7}\right)\right)=V_{\omega_{4}}, \quad I_{4}\left(\sigma\left(S_{8}\right)\right)=V_{\omega_{1}+\omega_{5}} \oplus V_{2 \omega_{8}}, \\
& I_{4}\left(\sigma\left(S_{9}\right)\right)=V_{\omega_{2}+\omega_{6}} \oplus V_{\omega_{1}+2 \omega_{9}} \oplus V_{\omega_{8}} \oplus V_{\omega_{6}} \oplus V_{\omega_{4}} \oplus V_{\omega_{0}} .
\end{aligned}
$$

The first of these statements is contained in [13, Theorem 1.3], where it is proved that $V_{\omega_{4}}$ generates the full ideal $I\left(\sigma\left(S_{7}\right)\right)$.

Comparing the previous theorem with Theorem 3.11 in [12], we deduce: 
Corollary 4. The kernel of the restriction map $\mathbb{C}\left[\sigma\left(S_{+}\right)\right]_{4} \rightarrow \mathbb{C}\left[\tau\left(S_{+}\right)\right]_{4}$ is equal to

$$
\bigoplus_{\substack{p \geq q \geq 1 \\ p-q \text { even } \\ p+q>4}} V_{\theta_{n-2 p}+\theta_{n-2 q}}^{\vee} \oplus \bigoplus_{p>4} V_{\theta_{n}+\theta_{n-2 p}}^{\vee}
$$

We also remark that quartic equations are not always induced by cubic equations.

Proposition 7. There is a component $V_{\omega_{n-3}+\omega_{n-7}}^{\vee}$ inside $I_{4}\left(\sigma\left(S_{+}\right)\right)$, of multiplicity one, consisting in quartic equations which are not induced by cubics - hence generators of $I\left(\sigma\left(S_{+}\right)\right)$.

Proof. The tensor product formulas given in Section 5 imply that the tensor product of $V_{\omega_{n}+\omega_{n-2 p}}$ or $V_{\omega_{n-1}+\omega_{n-2 p-1}}$ by $\Delta_{+}$, for $p \geq 4$, does not contain any copy of $V_{\omega_{n-3}+\omega_{n-7}}$. Therefore the tensor product of $I_{3}\left(\sigma\left(S_{+}\right)\right)$by $\Delta_{+}^{\vee}$ cannot contain $V_{\omega_{n-3}+\omega_{n-7}}^{\vee}$, which implies our claim.

\section{$7 \quad$ Freudenthal varieties}

The spinor varieties belong to the family of Freudenthal varieties

$$
L G \subset \mathbb{P}\left(\wedge^{\langle n\rangle} \mathbb{C}^{2 n}\right), \quad G \subset \mathbb{P}\left(\wedge^{n} \mathbb{C}^{2 n}\right), \quad S_{+} \subset \mathbb{P}\left(\Delta_{+}\right)
$$

which share, especially for $n=3$, many remarkable properties $[7,10]$. Here we denoted $G=$ $G(n, 2 n)$ the usual Grassmannian, and $L G=L G(n, 2 n)$ the Lagrangian Grassmannian, in their respective Plücker embeddings. Moreover we restrict to the spinor varieties of type $D_{2 n}$. The varieties $L G, G, S_{+}$are then Hermitian symmetric spaces of the same rank $n$. In fact they can be considered as models of the same variety over the (complexified) normed algebras $\mathbb{R}, \mathbb{C}$ and $\mathbb{H}$. For $n=3$ there is even an exceptional Freudenthal variety $\mathbf{G}$ over the Cayley algebra $\mathbb{O}$, which is the unique compact Hermitian symmetric space of type $E_{7}$.

Every Freudenthal variety is easily seen to be 3-transitive, and this leads to analogs of the homogeneous spaces embeddings discussed after Proposition 1:

$$
\begin{array}{lll}
S p_{n} \hookrightarrow L G, & S p_{2 n} / G L_{n} \hookrightarrow L G \times L G, & S p_{2 n} / O_{n} \hookrightarrow L G \times L G \times L G, \\
G L_{n} \hookrightarrow G, & G L_{2 n} / G L_{n} \times G L_{n} \hookrightarrow G \times G, & G L_{2 n} / G L_{n} \hookrightarrow G \times G \times G, \\
S O_{n} \hookrightarrow S_{+}, & S O_{2 n} / G L_{n} \hookrightarrow S_{+} \times S_{+}, & S O_{2 n} / S p_{n} \hookrightarrow S_{+} \times S_{+} \times S_{+}, \\
E_{6} \hookrightarrow \mathbf{G}, & E_{7} / \mathbb{C}^{*} E_{6} \hookrightarrow \mathbf{G} \times \mathbf{G}, & E_{7} / F_{4} \hookrightarrow \mathbf{G} \times \mathbf{G} \times \mathbf{G} .
\end{array}
$$

Of course this is strongly reminiscent of Freudenthal's magic square and its higher rank generalizations [10].

Cubic equations of the secant variety of a Freudenthal variety $F \subset \mathbb{P} V_{\omega}$ can be described uniformly. In fact the decomposition of $S^{3} V_{\omega}$ is known, and there are two types of isotypic components:

1. Those whose highest weight does not appear among the weights of $V_{2 \omega} \otimes V_{\omega}$; as we have already noticed, the duals of these components must belong to $I_{3}(\sigma(F))$.

2. Those whose highest weight does appear among the weights of $V_{2 \omega} \otimes V_{\omega}$. In fact this tensor product turns out to be multiplicity free; we write it down as

$$
V_{2 \omega} \otimes V_{\omega}=\bigoplus_{i} V_{\omega+\Omega_{i}}
$$


Let us discuss the case of $G=G(n, 2 n)$, the usual Grassmannian. Here $U^{\vee}=\wedge^{n} \mathbb{C}^{2 n}$, and the decomposition of $S^{3} U$ follows from the computations of Chen, Garsia and Remmel [2]. In fact these authors compute the plethysm $S^{3}\left(S^{n}\right)$ rather than $S^{3}\left(\wedge^{n}\right)$, but its is known that $S^{3}\left(\wedge^{n}\right)$ is dual to $S^{3}\left(S^{n}\right)$ for $n$ even; for $n$ odd $S^{3}\left(\wedge^{n}\right)$ is dual to $\wedge^{3}\left(S^{n}\right)$, which is also computed in [2]. Here by "dual", we mean that the highest weights of the irreducible components are coded by dual partitions, with the same multiplicities. This yields:

$$
S^{3}\left(\wedge^{n}\right)=\bigoplus_{\substack{a, b \leq n \\ a \leq 2 b, b \leq 2 a}} m_{a, b} V_{\omega_{n-a}+\omega_{n+a-b}+\omega_{n+b}},
$$

where the multiplicity $m_{a, b}$ is given by the following rule. If $b \geq a$, then $m_{a, b}=\left\lfloor\frac{2 a-b+1}{6}\right\rfloor$ if $2 a-b=1 \bmod 6$ or $a$ and $b$ are both even. Otherwise $m_{a, b}=\left\lceil\frac{2 a-b+1}{6}\right\rceil$. If $a>b$, then $m_{a, b}=\left\lfloor\frac{2 b-a+1}{6}\right\rfloor$ if $2 b-a=1 \bmod 6$ or $a$ and $b$ are both even. Otherwise $m_{a, b}=\left\lceil\frac{2 b-a+1}{6}\right\rceil$.

Observe that the decomposition of $S^{3}\left(\wedge^{n}\right)$ is thus notably more complicated that the decomposition of $S^{3} \Delta_{+}$. But most components, more precisely all those for which $a \neq b$, will automatically vanish on $\sigma(G)$, since

$$
\left(\wedge^{n}\right)^{(2)} \otimes \wedge^{n}=\bigoplus_{a \leq n} V_{\omega_{n-a}+\omega_{n}+\omega_{n+a}} .
$$

This indicates that we should pay special attention to the multiplicities $m_{a}:=m_{a, a}$, which are given by the formula

$$
m_{6 r+s}=r+1-\delta_{s, 1}, \quad r \geq 0, \quad 0 \leq s \leq 5 .
$$

Let us compute the generating series of these multiplicities:

$$
\begin{aligned}
\sum_{k \geq 0} m_{k} x^{k} & =\left(1+x+x^{2}+x^{3}+x^{4}+x^{5}\right) \sum_{r \geq 0}(r+1) x^{6 r}-\sum_{r \geq 0} x^{6 r+1} \\
& =\left(1+x+x^{2}+x^{3}+x^{4}+x^{5}\right)\left(1-x^{6}\right)^{-2}-x\left(1-x^{6}\right)^{-1} \\
& =\frac{1}{1-x^{6}}\left(\frac{1}{1-x}-x\right)=\frac{1}{1-x^{6}} \frac{1-x+x^{2}}{1-x} \\
& =\frac{1}{1-x^{6}} \frac{1+x^{3}}{1-x^{2}}=\frac{1}{\left(1-x^{2}\right)\left(1-x^{3}\right)} .
\end{aligned}
$$

This is the rational function we already met in Theorem 2!

One can check that the same phenomenon holds for the Lagrangian Grassmannian. We finally get the uniform statement:

Theorem 5. Let $X \subset \mathbb{P}\left(V_{\omega}\right)$ be a Freudenthal variety of rank $n$. Then there exist dominant weights $\Omega_{0}=2 \omega, \Omega_{1}, \ldots, \Omega_{n}$ such that:

1. for any $k \leq l$, one has

$$
V_{k \omega} \otimes V_{l \omega}=\bigoplus_{0 \leq i_{1} \leq \cdots \leq i_{k} \leq n} V_{(l-k) \omega+\Omega_{i_{1}}+\cdots+\Omega_{i_{k}}},
$$

in particular

$$
V_{\omega} \otimes V_{\omega}=\bigoplus_{i=0}^{n} V_{\Omega_{i}}, \quad V_{2 \omega} \otimes V_{\omega}=\bigoplus_{i=0}^{n} V_{\omega+\Omega_{i}}
$$


2. the multiplicity $m_{i}$ of $V_{\omega+\Omega_{i}}$ inside $S^{3} V_{\omega}$ is given by the generating series

$$
\sum_{i \geq 0} m_{i} x^{i}=\frac{1}{\left(1-x^{2}\right)\left(1-x^{3}\right)}
$$

3. the degree three part of $\mathbb{C}[\sigma(X)]$ is

$$
\mathbb{C}[\sigma(X)]_{3}=\bigoplus_{i \neq 1} V_{\omega+\Omega_{i}}^{\vee}
$$

Explicitly, the weights $\Omega_{i}$ are the following:

$$
\Omega_{i}= \begin{cases}2 \omega_{n-i} & \text { in type } C_{n}, \\ \omega_{n-i}+\omega_{n+i} & \text { in type } A_{2 n-1}, \\ \theta_{2 n-2 i} & \text { in type } D_{2 n} .\end{cases}
$$

\section{A toy case}

It may be interesting to observe that the list of Freudenthal varieties can be completed by two "degenerate" cases,

$$
V=v_{n}\left(\mathbb{P}^{1}\right) \subset \mathbb{P}\left(S^{n} \mathbb{C}^{2}\right), \quad P=\left(\mathbb{P}^{1}\right)^{n} \subset \mathbb{P}\left(\left(\mathbb{C}^{2}\right)^{\otimes n}\right) .
$$

The complete series $V, P, L G, V, S_{+}$, for a given $n$, has in particular the nice property that each variety is a (special) linear section of the next variety in the series.

The case of the rational normal curve $V$ is the simplest one. Its secant varieties are well understood (see e.g. [6, Part I, 1.3]). In particular the first secant variety has a nice desingularization in terms of the secant bundle. For simplicity let $U=\mathbb{C}^{2}$. On the projective plane $\mathbb{P}^{2}=\mathbb{P}\left(S^{2} U\right)$, the secant bundle is the rank two vector bundle $E$ defined by the exact sequence

$$
0 \rightarrow S^{n-2} U^{\vee} \otimes \mathcal{O}_{\mathbb{P}^{2}}(-1) \rightarrow S^{n} U^{\vee} \otimes \mathcal{O}_{\mathbb{P}^{2}} \rightarrow E \rightarrow 0
$$

Obviously $E$ is generated by global sections and $H^{0}\left(\mathbb{P}^{2}, E\right)=S^{n} U^{\vee}$. The line bundle $\mathcal{O}_{E}(1)$ defines a morphism $\eta$ from the threefold $\mathbb{P}(E)$ to $\mathbb{P}\left(H^{0}\left(\mathbb{P}^{2}, E\right)^{\vee}\right)=\mathbb{P}\left(S^{n} U\right)$. The next lemma is contained in $[8$, Section 3].

Lemma 7. The map $\eta: \mathbb{P}(E) \rightarrow \mathbb{P}\left(S^{n} U\right)$ is a desingularization of $\sigma(V)$. It induces an isomorphism

$$
\mathbb{C}[\sigma(V)]_{k} \simeq H^{0}\left(\mathbb{P}(E), \mathcal{O}_{E}(k)\right)=H^{0}\left(\mathbb{P}^{2}, S^{k} E\right) .
$$

Since $S^{k} E$ has no higher cohomology, as easily follows from (1), we can compute $H^{0}\left(\mathbb{P}^{2}, S^{k} E\right)$ $=\chi\left(\mathbb{P}^{2}, S^{k} E\right)$ as a (virtual) $G L(U)$-module, by using the equivariant localization formula of Atiyah-Bott [1]. In order to state the result we need a few more notations. We consider a maximal torus $T$ in $G L(U)$, and a compatible basis $(e, f)$ of $U$. We denote by $x, y$ the corresponding characters of $T$. For $g \in T$, the formula reads

$$
\operatorname{Trace}\left(g, \chi\left(\mathbb{P}^{2}, S^{k} E\right)\right)=\sum_{p \in\left(\mathbb{P}^{2}\right)^{T}} \frac{\operatorname{Trace}\left(g, S^{k} E_{p}\right)}{\operatorname{det}\left(1-g^{-1}, T_{p} \mathbb{P}^{2}\right)} .
$$


Here $\left(\mathbb{P}^{2}\right)^{T}$ denotes the set of fixed points of the action of $T$ on $\mathbb{P}^{2}$, which are the three lines in $S^{2} U$ generated by $e^{2}, e f, f^{2}$. In order to get a compact formula, we introduce an indeterminate $t$ and define

$$
P_{n}(t, x, y)=\sum_{k \geq 0} t^{k} \operatorname{Trace}\left(g, \chi\left(\mathbb{P}^{2}, S^{k} E\right)\right) .
$$

Let us compute this in from the Atiyah-Bott localization formula. At the fixed point $p=\left[e^{2}\right]$, the fiber of $E$ is the space of degree $n$ polynomials modded out by those divisible by $e^{2}$. A $T$-eigenbasis of $E_{p}$ is thus given by the images of $f^{n}, e f^{n-1}$. The induced $T$-eigenbasis of $S^{k} E_{p}$ consists in the degree $k$ monomials in $e, f$, multiplied by $f^{k(n-1)}$. Moreover, the $T$-module $T_{p} \mathbb{P}^{2}=\operatorname{Hom}\left(\left\langle e^{2}\right\rangle,\left\langle e f, f^{2}\right\rangle\right)$ has weights $y / x$ and $y^{2} / x^{2}$. The same straightforward analysis at the two other fixed points yields the formula

$$
\operatorname{Trace}\left(g, \chi\left(\mathbb{P}^{2}, S^{k} E\right)\right)=\frac{y^{k(n-1)} h_{k}(x, y)}{\left(1-\frac{x}{y}\right)\left(1-\frac{x^{2}}{y^{2}}\right)}+\frac{x^{k(n-1)} h_{k}(x, y)}{\left(1-\frac{y}{x}\right)\left(1-\frac{y^{2}}{x^{2}}\right)}+\frac{h_{k}\left(x^{n}, y^{n}\right)}{\left(1-\frac{x}{y}\right)\left(1-\frac{y}{x}\right)},
$$

where $h_{k}(x, y)$ denotes the sum of degree $k$ monomials in $x, y$. Summing over $k$, we get the closed expression $P_{n}(t, x, y)=P_{n}^{\prime}(t, x, y) /(x-y)\left(x^{2}-y^{2}\right)$, where

$$
P_{n}^{\prime}(t, x, y)=\frac{x^{3}}{\left(1-t x^{n}\right)\left(1-t x^{n-1} y\right)}+\frac{y^{3}}{\left(1-t y^{n}\right)\left(1-t x y^{n-1}\right)}-\frac{x y(x+y)}{\left(1-t x^{n}\right)\left(1-t y^{n}\right)} .
$$

Extracting the coefficient of $t^{3}$, we get the character $P_{n}^{3}(x, y)=Q_{n}^{3}(x, y) /(x-y)$ of the $G L(U)$ module $\left.H^{0}\left(\mathbb{P}^{2}, S^{3} E\right)\right)=\mathbb{C}[\sigma(V)]_{3}$, with

$$
Q_{n}^{3}(x, y)=x^{2 n}\left(x^{n+1}+x^{n-1} y^{2}+\cdots+x y^{n}\right)-y^{2 n}\left(x^{n} y+\cdots+x^{2} y^{n-1}+y^{n+1}\right) .
$$

Since the character of the $G L(U)$-module $S^{p} U \otimes(\operatorname{det} U)^{q}$ is $\left(x^{p+q+1} y^{q}-x^{q} y^{p+q+1}\right) /(x-y)$, this means that

$$
\mathbb{C}[\sigma(V)]_{3}=S^{3 n} U \oplus \bigoplus_{k=2}^{n} S^{3 n-2 k} U \otimes(\operatorname{det} U)^{k} .
$$

This is in agreement with Theorem 5 , for $\omega=n \omega_{1}$, but taken with a grain of salt. Indeed, all the formulas in Theorem 5 are correct, with

$$
\Omega_{i}=2 \omega-2 i \omega_{1},
$$

except for the first one for $k \geq 2$. Indeed, the weights $\Omega_{i}$, in the special case we are dealing with, are not linearly independent (recall that this is a degenerate case!). To get a correct result from the first formula of Theorem 5 (which is just an instance of the Clebsch-Gordan formula), one needs to correct this by taking all non zero multiplicities to the value one.

One can go further and compute the character $P_{n}^{k}(x, y)=Q_{n}^{k}(x, y) /(x-y)$ of the $G L(U)$ module $H^{0}\left(\mathbb{P}^{2}, S^{k} E\right)=\mathbb{C}[\sigma(V)]_{k}$. Indeed, if $q_{n, s}^{k}$ is the coefficient of $x^{n k+1-s} y^{s}$ in this polynomial, one can deduce for $n k \geq 2 s$ the induction formula

$$
q_{n, s}^{k}-q_{n, s-2}^{k}=\delta_{s \leq k}-\delta_{n \mid s-1}-\delta_{n \mid s-2} .
$$

This leads to the following statement:

Proposition 8. Let $\epsilon \in\{0,1\}$ be the parity of $s$, with $n k \geq 2 s$. The multiplicity of $S^{k n-2 s} U \otimes$ $(\operatorname{det} U)^{s}$ inside $\mathbb{C}[\sigma(V)]_{k}$ is

$$
q_{n, s}^{k}=\min \left(\left\lfloor\frac{s}{2}\right\rfloor,\left\lfloor\frac{k-\epsilon}{2}\right\rfloor\right)-\left\lfloor\frac{s-1}{n}\right\rfloor .
$$


For example, for $k=4$ one gets that the multiplicity $q_{n, s}^{4}$ is equal to 0 for $s=1$, to 2 for $s$ even and $4 \leq s \leq 2 n$, and to 1 otherwise. For $n=3$ the secant variety is the whole of $\mathbb{P}^{3}$, and one recovers the formulas of Chen-Garsia-Remmel for the symmetric powers of $S^{3} \mathbb{C}^{2}$.

For the tangent surface $\tau(V)$ to the normal rational curve we have

$$
\mathbb{C}[\tau(V)]_{k}=\bigoplus_{\substack{s \leq k \\ s \neq 1}} S^{k n-2 s} U \otimes(\operatorname{det} U)^{s} .
$$

We recover the fact that we have already observed for the spinor varieties: $\tau(V)$ has, for $n \geq 4$, and contrary to $\sigma(V)$, some non trivial cubic equations.

It is tempting to imagine that very similar formulas could hold for the other types of Freudenthal varieties. Observe, nevertheless, that the ideal of $\sigma(V)$ is generated by cubics (obtained as $3 \times 3$ minors of catalecticant matrices [8]). We have seen that this statement is not true for spinor varieties.

\section{Acknowledgments}

I thank J.M. Landsberg, G. Ottaviani and J. Weyman for useful discussions.

\section{References}

[1] Atiyah M.F., Bott R., A Lefschetz fixed point formula for elliptic complexes. II. Applications, Ann. of Math. (2) 88 (1968), 451-491.

[2] Chen Y.M., Garsia A.M., Remmel J., Algorithms for plethysm, in Combinatorics and Algebra (Boulder, Colo., 1983), Contemp. Math., Vol. 34, Amer. Math. Soc., Providence, RI, 1984, 109-153.

[3] Chevalley C., The algebraic theory of spinors and Clifford algebras, Collected works, Vol. 2, Springer-Verlag, Berlin, 1997.

[4] Dress A.W.M., Wenzel W., A simple proof of an identity concerning Pfaffians of skew symmetric matrices, Adv. Math. 112 (1995), 120-134.

[5] Howe R., Tan E.C., Willenbring J.F., Stable branching rules for classical symmetric pairs, Trans. Amer. Math. Soc. 357 (2005), 1601-1626, math.RT/0311159.

[6] Iarrobino A., Kanev V., Power sums, Gorenstein algebras, and determinantal loci, Lecture Notes in Mathematics, Vol. 1721, Springer-Verlag, Berlin, 1999.

[7] Kaji H., Yasukura O., Projective geometry of Freudenthal's varieties of certain type, Michigan Math. J. 52 (2004), 515-542.

[8] Kanev V., Chordal varieties of Veronese varieties and catalecticant matrices, J. Math. Sci. (New York) 94 (1999), 1114-1125, math.AG/9804141.

[9] Knuth D.E., Overlapping Pfaffians, Electron. J. Combin. 3 (1996), no. 2, paper 5, 13 pages, math.CO/9503234.

[10] Landsberg J.M., Manivel L., The projective geometry of Freudenthal's magic square, J. Algebra 239 (2001), 477-512, math.AG/9908039.

[11] Landsberg J.M., Manivel L., On the ideals of secant varieties of Segre varieties, Found. Comput. Math. 4 (2004), 397-422, math.AG/0311388.

[12] Landsberg J.M., Weyman J., On tangential varieties of rational homogeneous varieties, J. Lond. Math. Soc. (2) 76 (2007), 513-530, math.AG/0509388.

[13] Landsberg J.M., Weyman J., On secant varieties of compact Hermitian symmetric spaces, arXiv:0802.3402.

[14] LiE, A computer algebra package for Lie group computations, available at http://young.sp2mi.univ-poitiers.fr/ marc/LiE/.

[15] Zak F.L., Tangents and secants of algebraic varieties, Translations of Mathematical Monographs, Vol. 127, American Mathematical Society, Providence, RI, 1993. 